\title{
Stress optimization and study of the sensitivity to geometric variations of a spur gear tooth profile
}

\author{
David Guyonneau $^{1,2, a}$, Emmanuel Mermoz ${ }^{2}$, Jean Mailhe ${ }^{1}$, Jean-Michel Sprauel ${ }^{1}$ \\ and Jean-Marc Linares ${ }^{1}$ \\ 1 Aix Marseille Université, CNRS, ISM UMR 7287, 13288, Marseille Cedex 09, France \\ 2 EUROCOPTER, Aéroport Internationale Marseille Provence, 13725 Marignane, France
}

\begin{abstract}
This paper presents an approach for obtaining an optimized geometry for the flank of a tooth by minimizing the equivalent contact stress. The stress calculation method is based on Hertz theory. As the majority of tooth profiles are involute, the geometric variation of the flank of the tooth is achieved variationally relative to the involute profile. The optimum profile is obtained by Monte Carlo simulation. During this optimization, a polynomial expression of the tooth geometry is used. The parameters influencing the simulation are the four characteristic contact points. The approach presented has been applied in a representative case. A study of the geometric sensitivity was conducted on the optimized tooth profile. Two different approaches were considered: by Monte Carlo simulation and analytical propagation. The robust and linear nature of the behavior of the tooth profile was demonstrated when it was subjected to geometric variations.
\end{abstract}

Key words: Spur Gear / Tooth profile / Tooth Contact Analysis (T.C.A.) / Monte Carlo simulation / Uncertainty

\begin{abstract}
Résumé - Optimisation en contrainte et étude de sensibilité aux variations géométriques d'un profil de denture d'engrenage. Cet article propose une démarche permettant d'obtenir une géométrie optimisée d'un flanc de denture par la minimisation de la contrainte équivalente au contact. La méthode de calcul de la contrainte est fondée sur la théorie généralisée de Hertz. Comme la majorité des profils de denture sont en développante de cercle, l'évolution géométrique du flanc de denture est réalisée de manière variationnelle par rapport au profil en développante de cercle. Le profil optimal est obtenu par une simulation de Monte Carlo. Lors de cette optimisation, une expression polynomiale de la géométrie de la denture a été utilisée. Les paramètres pilotant la simulation sont les quatre points caractéristiques de contact. La démarche proposée a été mise en œuvre sur un cas représentatif. Sur le profil de denture optimisé, une étude de sensibilité géométrique a été menée. Deux approches ont été envisagées : par simulation de Monte Carlo et par la méthode de propagation analytique. La robustesse et la linéarité du comportement du profil de denture soumis à des variations géométriques sont démontrées.
\end{abstract}

Mots clés : Engrenages cylindriques / Profil de denture / Tooth Contact Analysis (T.C.A.) / Simulation de Monte Carlo / Incertitudes

\section{Introduction}

In the aeronautical sector, the mechanical transmission of power is achieved through various gear configurations (Fig. 1). Full control of the geometry of tooth

a Corresponding author: david.guyonneau@eurocopter .com profiles and their manufacture are indispensible in order to guarantee the reliability of the mechanism. In order to ensure the correct operation of a reduction gearbox, the majority of studies examine the mechanical strength of the gears under load. For helicopter manufacturers, the search for performance involves minimizing weight whilst increasing the efficiency and the service life of the mechanical components constituting, for example, the Main 


\section{Nomenclature}

\begin{tabular}{|c|c|}
\hline \multicolumn{2}{|r|}{ Latin Letters } \\
\hline $\mathrm{A}, \mathrm{B}, \mathrm{C}, \mathrm{D}$ & Contact characteristic points \\
\hline$a, b, c, d$ & Tooth flank characteristic points \\
\hline$C_{1}$ & Input torque \\
\hline Cix, Ciy & Polynomial coefficients \\
\hline $\boldsymbol{f}(\theta)$ & Polynomial function \\
\hline$F_{\mathrm{C}}$ & Contact load \\
\hline I & Primitive point \\
\hline$n_{\text {contact }}$ & Number of contacts \\
\hline$P_{1}$ & Input power \\
\hline$R_{\mathrm{pi}}$ & Primitive radius \\
\hline$R_{\mathrm{bi}}$ & Basis radius \\
\hline$R_{\mathrm{ti}}$ & Head radius \\
\hline$W$ & Range \\
\hline$x_{i}$ & $X$ coordinate \\
\hline$y_{i}$ & $Y$ coordinate \\
\hline$z_{i}$ & $Z$ coordinate \\
\hline$\left(x_{i}, y_{i}, z_{i}\right)$ & Cartesian reference system \\
\hline$Z_{i}$ & Tooth number \\
\hline \multicolumn{2}{|r|}{ Greek letters } \\
\hline$\alpha$ & Pressure angle \\
\hline$\varepsilon_{t}$ & Total contact ratio \\
\hline$\mu$ & Friction coefficient \\
\hline$\theta$ & Angular variable \\
\hline$\omega_{1}$ & Input rotation speed \\
\hline$\sigma_{\text {eq }}$ & Equivalent stress \\
\hline$\sigma$ & Standard deviation \\
\hline \multicolumn{2}{|r|}{ Subscripts } \\
\hline$i$ & Refers to element $i$ \\
\hline 1 & Refers to the pinion \\
\hline 2 & Refers to the gear \\
\hline$S$ & Refers to contact input (Start) \\
\hline$E$ & Refers to contact output (End) \\
\hline
\end{tabular}

Gearboxes (M.G.B.). A 300 kg M.G.B., including 5 reduction stages, transmitting two megawatts power is shown in Figure 1.

Currently, the sizing and geometric design of meshing surfaces constitute complex strategic domains. In the field of application to helicopter power gearboxes, specific work has been undertaken to extend the service life of gear assemblies and to ensure improved behavior under load. With respect to this problem, work is essentially focused on:

- characterization of the material and its various treatments (shot peening, nitriding),

- elaboration of behavioral laws representing the contact physics,

- geometric modification evolution of the meshing surfaces.

This paper concerns the third item. In this mechanical environment, the scientific literature proposes numerous studies; a representative sample will be presented in the bibliographical study (Sect. 2). Two study paths are examined in this paper: presentation of a method for generating a tooth profile minimizing the equivalent contact stress during meshing and a study of the sensitivity of this

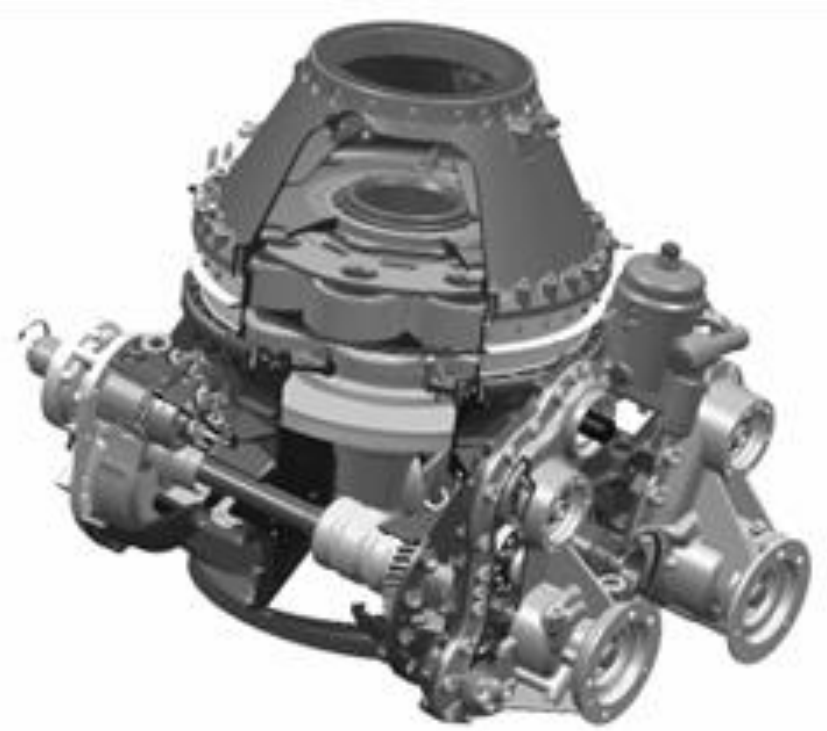

Fig. 1. Helicopter main gearbox, global view of gears.

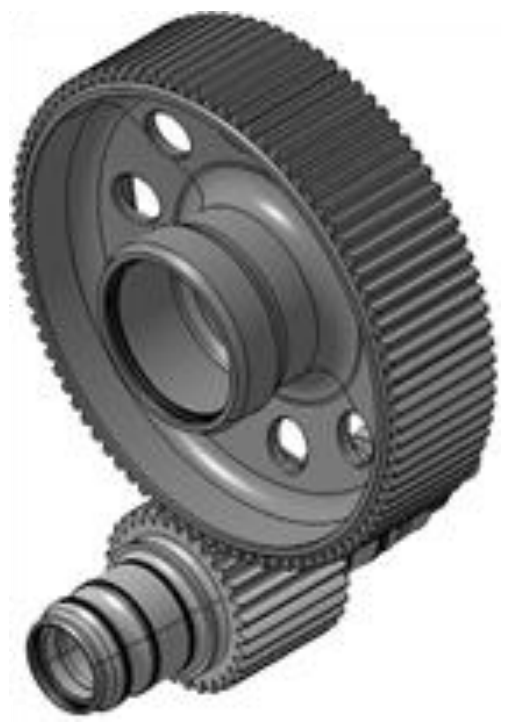

Fig. 2. Involute spur gear example.

tooth flank to geometric variations. The optimization approach is explained in the third paragraph of this paper. It looks exclusively at external spur gears. The geometric and mechanical characteristics of the gear shown in Figure 2 are detailed in the remainder of the document (Sect. 3).

\section{Bibliography on the geometric generation and sensitivity of gears}

Among the literature, the work by Henriot [1] is a considerable source of information on the design of gears and the characterization of their fields of use. It presents the usual definition of the variables allocated to gears. The work by Henriot [1] incidentally contributed to the 
elaboration and compilation of international standards (ISO standard 6336 in particular). The involute tooth profile represents the founding basis for modern gear geometries. The sizing of the teeth is achieved by accounting for the spatial requirements, calculating the contact pressure (pitting) related to the applied loads, estimating the maximum shear stress in the root of the tooth (fracture), minimizing the gearing noise and optimizing the efficiency.

This design environment enables definition of the functional operating conditions of a gear. Our bibliography hinges around three main axes:

- the methods for obtaining tooth profiles,

- a study of the mechanical behavior based on the equivalent contact stress,

- the influence of geometric variations of the flank of the tooth.

\subsection{Methods for generating tooth profiles}

The notion of conjugated profiles is an inescapable aspect in the theory of gears. ISO standard 21771 [2] specifies the concept and the geometry of tooth flank. It gives some advice to modify the local tooth profile in order to improve the meshing. The paper by Spitas et al. [3] proposes a method for obtaining combined tooth profiles using the properties of the involute. The work by Ye \& Ye [4] and Simon [5] also studies the geometry and the methods for obtaining a gear tooth profile. They introduce local geometric modifications in order to observe their influence on the behavior of the gear during meshing. Faggioni et al. [6] optimize the geometry of spur gears in order to improve their dynamic behavior. For that purpose, they construct a rheological model using springs and dampers. All these works propose a geometric definition for the flank of a tooth. The paper of Velex et al. [7] and Ghribi et al. [8] purposed also local geometrical modifications of the gear tooth profile. They studied the influence of profile modifications on transmission errors.

\subsection{Characterization of the loading}

A great number of studies were performed to characterize the load applied to gears. Velex et al. [7] used an analytical model to define the mechanical solicitations applied to modify spur and helical gear tooth profiles. In similar approach, Pedrero et al. [9] studied an involute tooth profile of an external spur gear under load by characterization of the mechanical behavior along the line of action. The characterization of the stresses in gearboxes has been covered by Osman and Velex [10] and Pedrero et al. [11]. Hwang et al. [12] examine the identification and analysis of the equivalent contact stress. However, these numerous works [6-12] do not question the geometry of the involute tooth profile. They concentrate on characterization of the mechanical loading at the contact points. Most of these works are based on F.E.M. (Finite Element
Method) calculations, but some of them characterize the contact zone using Hertz theory. These various works consider the mechanical aspect of the problem by characterizing the loading on the tooth.

The work by Litvin et al. [13] covers the geometric modification of a tooth profile. Their paper has three objectives: to improve the contact, to reduce the transmission error and to characterize the equivalent contact stress. The search for an optimum profile by Litvin [13] is common to our paper. Nevertheless, the main difference lies in the methods for obtaining the tooth profile geometries. Indeed, in our paper, the proposed modification is variational relative to the involute without reference to known profiles. In the work by Litvin [13], it is based on involute and epicycloidal profiles. The variational aspect is important since it does not presuppose the optimum profile which results from the approach.

\subsection{Geometric sensitivity}

In the second part of this paper, a study was made of the sensitivity of tooth profiles to geometric variations. The work by Linares et al. [14] and by Zamponi et al. [15] on the geometric error of mechanical parts demonstrates the impact of loading on the geometric distortion of a mechanical system. In these papers, Zamponi et al. [16,17] examine the effect of local distortion (at the point of contact) as well as structural distortion (displacement of the shafts) on its mechanical systems. However, the geometric variations due to the manufacturing process are not considered. They propose original methods for calculating the pressure using analytical, numerical (F.E.M.) or hybrid methods using substitution elements.

The work by $\mathrm{Xu}$ et al. [18] proposes the estimation of gearbox performance according to the input conditions. These works concern cylindrical gears with straight or helical teeth. This study is based on a contact friction model and concludes with a numerical calculation (F.E.M.) of mechanical efficiency. Xu thus studies the performance of reduction gears according to the geometric design data, the operating conditions and the assembly and manufacturing errors.

In addition to the work by Xu et al. [18], two other papers examine sensitivity. These are the paper by Linares et al. [19] and that by Bruyère et al. [20]. Linares uses Monte Carlo simulation and Analytical Propagation. The work developed by Bruyère et al. [20], proposes a statistical analysis of the functional surfaces of a bevel gear. This sensitivity study looks at the impact of tolerance choices on the quality and manufacturing costs of the meshing surfaces of a reduction gear.

In conclusion, the objective of our work is to propose a new gear tooth profile which minimizes the equivalent contact stress, and to study the robustness under stress of this new geometry according to manufacturing errors. The use of error propagation methods enabled investigation of the robustness under stress of the optimized tooth profile according to the geometric variations due to the capabilities of the production means. 


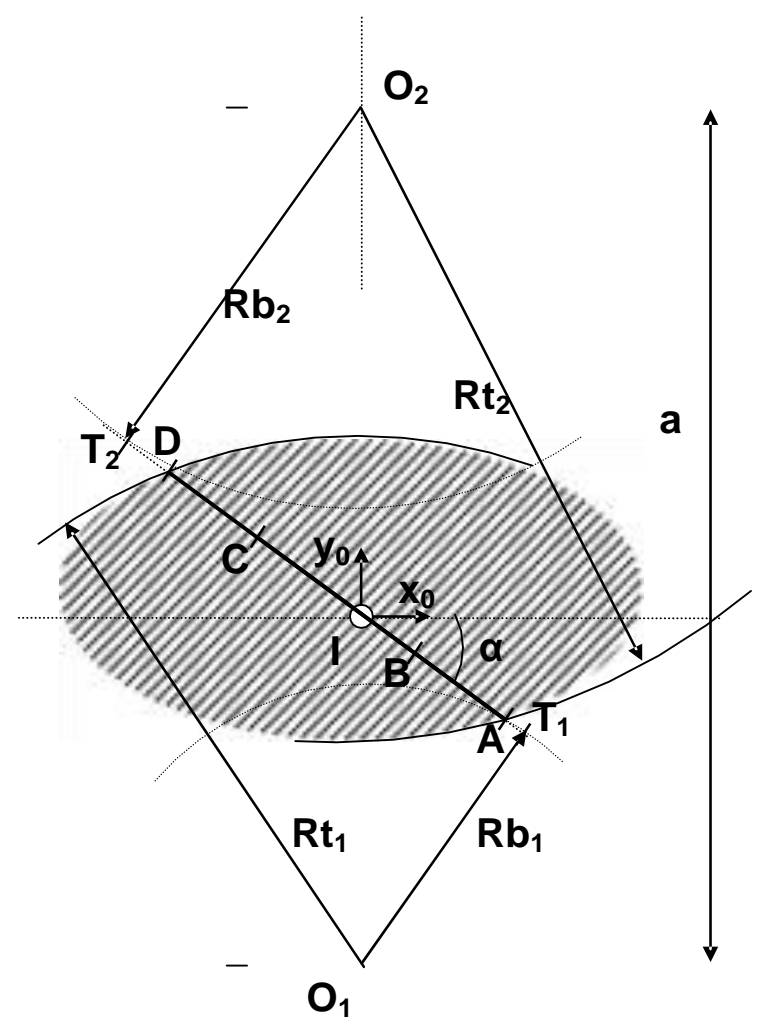

Fig. 3. Simplified geometry of the gear.

\section{Method for optimizing the tooth profile under stress}

The approach used demands the establishment of a simplified geometry of the gear (Fig. 3). In our resolution method, the gear is a spur gear. The potential zone of contact is shaded in Figure 3, and the line of contact, which is characteristic of an involute tooth profile, is defined by the segment between points A (input point) and $\mathrm{D}$ (output point). It is determined by the functional head radii of the pinion (indexed 1) and the wheel (indexed 2), and is inclined by the angle of pressure $\alpha$.

In the involute tooth profile configuration, the contact path must be included into [ $\left.T_{1} T_{2}\right]$ segment. The nominal localization of the contact entry point $\mathrm{A}$ is derived from a previous minimization of the maximum equivalent contact stress. The coordinate of point $\mathrm{D}$ is derived from the contact ratio. The basis pitch defines the contact distance between two successive teeth and permits to set points $B$ and $\mathrm{C}$ which correspond to the transition of one to two teeth under contact.

Only four inputs are required in the method developed in this paper:

- Pressure angle, “ $\alpha$ ”, which characterizes the angle of inclination with the line of contact.

- Distance between centers, " $a$ ", the distance between the centers of rotation, $\mathrm{O}_{1}$ and $\mathrm{O}_{2}$.
Table 1. Spur Gear Characteristics of the case study.

\begin{tabular}{cccc}
\hline Parameters & Abbreviations & Values & Units \\
\hline Center distance & $a$ & 150 & $\mathrm{~mm}$ \\
Number of teeth-pinion & $Z_{1}$ & 31 & $/$ \\
Number of teeth-wheel & $Z_{2}$ & 89 & $/$ \\
Module & $m$ & 2.5 & \\
Pressure angle & $\mathrm{mm}$ & & \\
Face width & $\alpha$ & $22^{\circ}$ & $\mathrm{deg}$ \\
Crowning in the width & $b$ & 65 & $\mathrm{~mm}$ \\
Contact ratio & $C_{y}$ & 0.01 & $\mathrm{~mm}$ \\
Power entrance & $\Phi_{\pi}$ & 19.00 & $\mathrm{kX}$ \\
Speed rotation entrance & $\omega_{1}$ & 23000 & rot.min \\
\hline
\end{tabular}

- Reduction ratio, “ $\boldsymbol{p}$ ', determined by the number of teeth on the pinion and the wheel (Eq. (1)), and

- Contact ratio, " $\varepsilon_{t}$ ", which is used to determine the distance between A and D in Figure 4 (Eq. (2)).

$$
i=(-1)^{n} \frac{Z_{1}}{Z_{2}}
$$

with $n=1$ for an external gear and $n=0$ for an internal gear.

$$
\varepsilon_{t}=\frac{\left(\theta_{E}-\theta_{S}\right)}{2 \pi} Z_{1}
$$

where $\theta_{S}, \theta_{E}$ are the angular positions of the pinion at input (Start: $S$ ) and output (End: E).

The pitch circle radius (Eq. (3)) and the basis radius (Eq. (4)) are directly linked to three of them.

$$
\underset{\substack{R_{\mathrm{bi} 1}=R_{\mathrm{pi}} \cos (\alpha)}}{\frac{i}{=}}
$$

However, ahesadparapater do sot suffice for the estab) lishment of the entire operating environment of the gear. In order to supplement this initialization data, some other characteristics are required, such as the width of the teeth $(b)$, the input power $\left(P_{1}\right)$ or the rotation speed of the pinion $\left(\omega_{1}\right)$ are required in order to establish the operating conditions of the gear considered. The characteristics of the gear studied are presented in Table 1.

Once these data has been defined, it is possible to progress to using the profile optimization method. The flowchart for this method is shown in Figure 4.

In this Figure 4, our method is principally composed of three steps. First, the initial configuration corresponding to a perfect involute tooth profile is considered. The in-depth stresses are computed using a generalized analytical Hertz contact method allowing to define the maximal equivalent stress during meshing. As a first approach, only the quasi-static behavior is considered. Secondly, a modified tooth profile is generated randomly using a Monte Carlo simulation algorithm. The in-depth stresses 


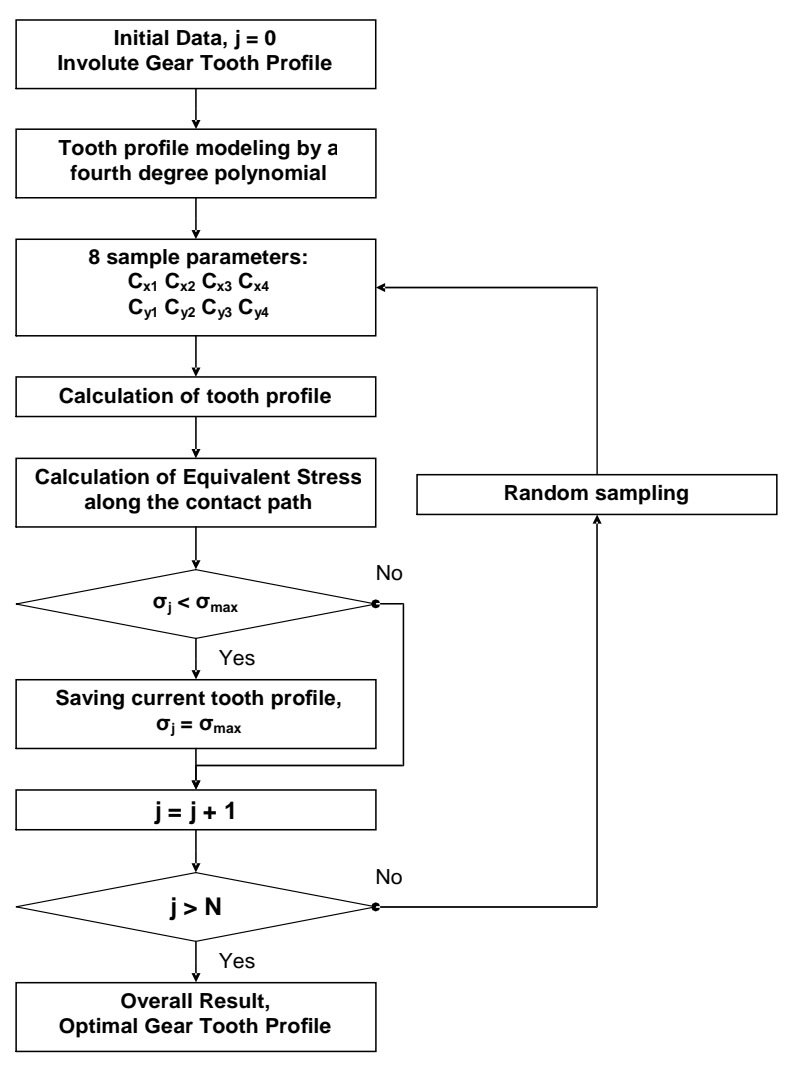

Fig. 4. Flowchart optimization method.

are then recomputed to the actual best solution. Only enhanced configuration is saved. At the end of the process, the optimized tooth profile parameters are displayed.

This procedure is detailed in the next paragraphs.

\subsection{Evolution of the tooth profiles}

The optimization procedure involves examining the contact during meshing. The geometric definition of the tooth profile meets the constraints of gear theory. These are essentially of two types, non-interference of the teeth and compliance with the conjugation of the profiles. The choice of modifying the profile variationally relative to involute enables partial avoidance of the constraints. There is no modification to the profile in the width of the tooth. The curvature $C_{y}$ in the width of the tooth is part of the input data provided in Table 1. This curvature is retained.

Equation (5) shows the expression of the involute tooth profile of the pinion, in Cartesian coordinates centered on pitch point I:

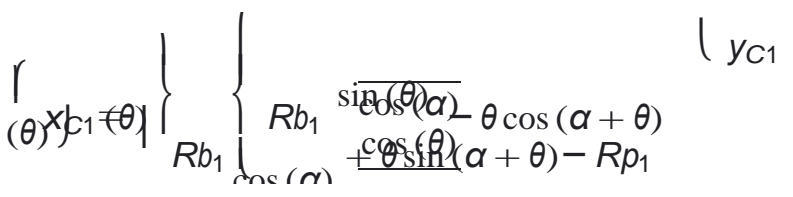

$$
\theta \in\left[\theta_{S} ; \theta_{E}\right]
$$

with $\theta_{S}, \theta_{E}$ the angular positions of the pinion at the contact input (point A) and output (point D).

$$
\begin{aligned}
& \left.\mid x_{C 2}(\theta)\right\}^{\prime}\left\{R b_{2} \frac{\sin (i \theta)+i \theta \cos (\alpha+i \theta)}{-\left(y_{C 2}\right.}\right. \\
& (\theta))^{\prime}=\left.\right|_{R b_{2}} \stackrel{\cos (i \theta)}{-\frac{\cos (\alpha)}{\cos (\alpha)}} \\
& \theta \in\left[\theta_{S} ; \theta_{E}\right]
\end{aligned}
$$

here, $i$ defines the reduction ratio.

These initial involute profiles for the pinion (Eq. (5)) and the wheel (Eq. (6)) are slightly modified. For their purpose, the initial functions are subsequently multiply by weighting functions. These functions are approximated by power series expansions. In a spur gear with a contact ratio between 1 and 2, there are four characteristic points during meshing: the input point (A), the output point (D) and two transition points from one to two teeth under contact (B and C). For this reason, the power series expansion functions were restricted to 4 th degree polynomials. This avoids excessive oscillations of the tooth profiles. The coefficients of the polynomials are selected randomly by Monte Carlo simulation (Sect. 3.3).

The only variable in these functions is the angular position of the pinion $\theta$. The analytical expression of the modified profile is proposed in Equation (7). The reasoning adopted is analogous for the geometric evolution of the wheel. In the remainder of this paper, the development of the approach and the presentation of results are provided only for the active profile of the pinion.

\section{see equation (7) next page.}

In these equations, angle $\theta$ is the only variable. For $\theta=0$, the point of the tooth profile is at I at the pitch radius. With this single variable, it is possible to reconstruct the tooth profile in $2 \mathrm{D}$. To do this, the extreme contact positions must be defined. As the contact ratio is defined, $\theta_{E}$ is deduced from $\theta_{S}(\mathrm{Eq}$. (2)).

\subsection{Calculation of the equivalent contact stress}

The geometric optimization of the tooth profiles is achieved by minimizing the equivalent contact stress in accordance with Von Mises criterion. To undertake this stress calculation during meshing of the pinion with the wheel, it is necessary to know the contact loading at any time.

The rheological data are defined by Young's modulus $E$ and Poisson's coefficient $v$ for the materials used. The loading is deduced from a nominal load, relative to the input power $P_{1}$, the rotational speed of the pinion $\omega_{1}$ and the contact radius at the time considered $R_{C}(\theta)$. The load $F(\theta)$ is distributed according to the number of teeth under contact $n_{\text {contact }}$. Isotorque sharing is selected for that purpose. This assumption permits fast search of the optimal solution. This best configuration is finally validated using F.E.M. modeling. This number is determined from the contact ratio and the current angular position 


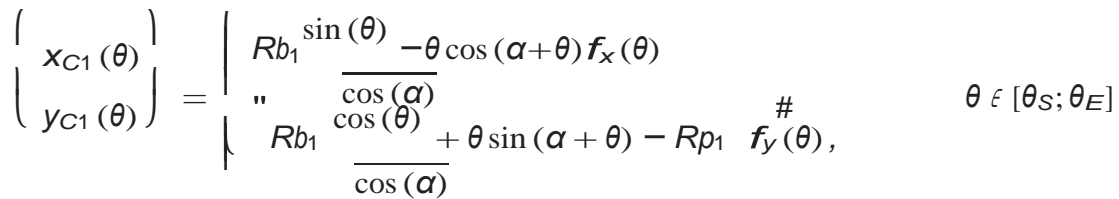

$$
\begin{aligned}
& \left\{\begin{array}{l}
x_{C 1}(\theta) \\
y_{C 1}(\theta)
\end{array}\right)=\left\{\begin{array}{ll}
R b_{1} \frac{\sin (\theta)}{\cos (\alpha)} \theta \cos (\alpha+\theta) 1+c_{1 x} \theta+c_{2 x} \theta^{2}+c_{3 x} \theta^{3}+c_{4 x} \theta^{4} \\
R b_{1} \frac{\cos (\theta)}{\cos (\alpha)} \theta \sin (\alpha+\theta)-R p_{1} 1+c_{1 y} \theta+c_{2 y} \theta^{2}+c_{3 y} \theta^{3}+c_{4 y} \theta^{4},
\end{array} \quad \theta \in\left[\theta_{S} ; \theta_{E}\right]\right.
\end{aligned}
$$

of the tooth profile (Eq. (8)). Generally, the contact ratio is between 1 and 2, thus:

$$
\begin{aligned}
& \mid \theta(r d) \in \theta_{E} ; \theta_{S}-\frac{2 \pi}{Z_{1}} \theta_{E}+\frac{2 \pi}{Z_{S}} \theta_{Z_{1}} \Rightarrow n_{\text {contact }}=2 \\
& \theta(r d) \epsilon \quad \theta_{S}-\frac{2 \pi}{Z} ; \theta_{E}+\frac{2 \pi}{Z_{1}} \Rightarrow n_{\text {contact }}=11
\end{aligned}
$$

Knowing the input torque $C_{1}$ (Eq. (9)) and the number of teeth in contact $n_{\text {contact }}$, the load evenly distributed between the teeth can be obtained according to $\theta$ (Eq. (10)).

$$
\begin{aligned}
C_{1} & =\frac{P_{1}}{\omega_{1}} \\
F(\theta) & =\frac{C_{1}}{n_{\text {contact }}(\theta) R_{C}(\theta)}
\end{aligned}
$$

The meshing simulation enables observation of the contact between the pinion and the wheel. The characterization of the contact is expressed in 100 angular positions of one pinion tooth. These 100 positions are ranged between $\theta=\frac{-\pi}{Z_{1}}$ and $\theta=\frac{\pi}{Z_{1}}$.

In Figure 5, the calculation of the equivalent curvature is an interesting intermediate result for two reasons.

First, the curvature only depends on the geometry of the profiles under contact. The equivalent curvature at the point of contact is related to the angular position of the pinion. Secondly, this equivalent curvature is involved in the calculation for the equivalent contact stress (Fig. 6).

In order to calculate the equivalent curvature, it is necessary to know the curvature, at the contact point of the pinion and the wheel. Knowing the equation of profile considered (Eq. (7)), the analytical expression for the curvature (Eq. (11)) is obtained by derivation.

$$
\gamma_{C 1}(\theta)= \pm \frac{x_{C 1}^{\theta}(\theta) y_{C 1}^{\theta 0}(\theta)-y_{C 1}^{0}(\theta) x_{C 1}^{00}(\theta)}{\left(x_{C 1}^{\theta}(\theta)\right)^{2}+\left(y_{C 1}^{0}(\theta)\right)^{2^{3 / 2}}}
$$

The curvature of the wheel is calculated similarly from the expression of its tooth profile. Knowing the curvature of the pinion and the wheel, it is easy to calculate the equivalent curvature at each point of contact (Eq. (12)). Equation (12) shows the expression for this parameter.

$$
Y_{\text {ceq }}(\theta)=Y_{C 1}(\theta)+Y_{C 2}(\theta)
$$

The calculation of the maximum equivalent stress in the sub-layer is established for all angular contact positions. In Figure 6, the equivalent stresses of the initial involute profile and the optimized profile are presented according to the angular position of the pinion. The 4 characteristic points $\mathrm{A}, \mathrm{B}, \mathrm{C}$ and $\mathrm{D}$, of the mesh are identified.

Variable $\theta$ characterizes the angular position of the pinion. It is the common variable between the expression for the contact stress and the equation for the associated tooth profile. The Monte Carlo simulation modifies the geometry of the tooth profile to achieve an optimized profile. The procedure selects the best profile. A summary of the Monte Carlo simulation parameters is provided in next paragraph.

\subsection{Optimization}

In the search for the optimum profile, only the maximum equivalent Von Mises stress by Hertz contact is examined along the flank of the teeth (Sect. 3.2). In fact, the profile providing the most benefit is adopted. The coefficients of the tooth profile are picked randomly by Monte Carlo simulation. The number of samples " $N$ " of the Monte Carlo simulation is a parameter to be defined by the designer. The aim is to obtain the parametric equation of tooth profiles which minimizes the equivalent contact stress. Thus the variation ranges of the coefficients are simulation parameters. In this Monte Carlo simulation, apart from the random picking of coefficients for the polynomial equation of the tooth flanks, the main parameter to be defined concerns the number of samples. Several tests have been carried out to define the value providing the best stress benefits. The result of this study is shown in Figure 7.

An estimate of the optimum number of samples for stress has been conducted at points $\mathrm{B}$ and $\mathrm{C}$ where the stress is at its maximum. Indeed, these two points are the most constraining in terms of the mechanical strength of the teeth. This is explained by the fact that there is just one tooth in contact between these two points. In Figure 7, the benefit ratio relative to the initial involute stress is represented for different numbers of samples. A number of 20000 samples has been chosen.

All the criteria for the tooth profile optimization procedure are shown. Next paragraph shows and comments on the results obtained. 


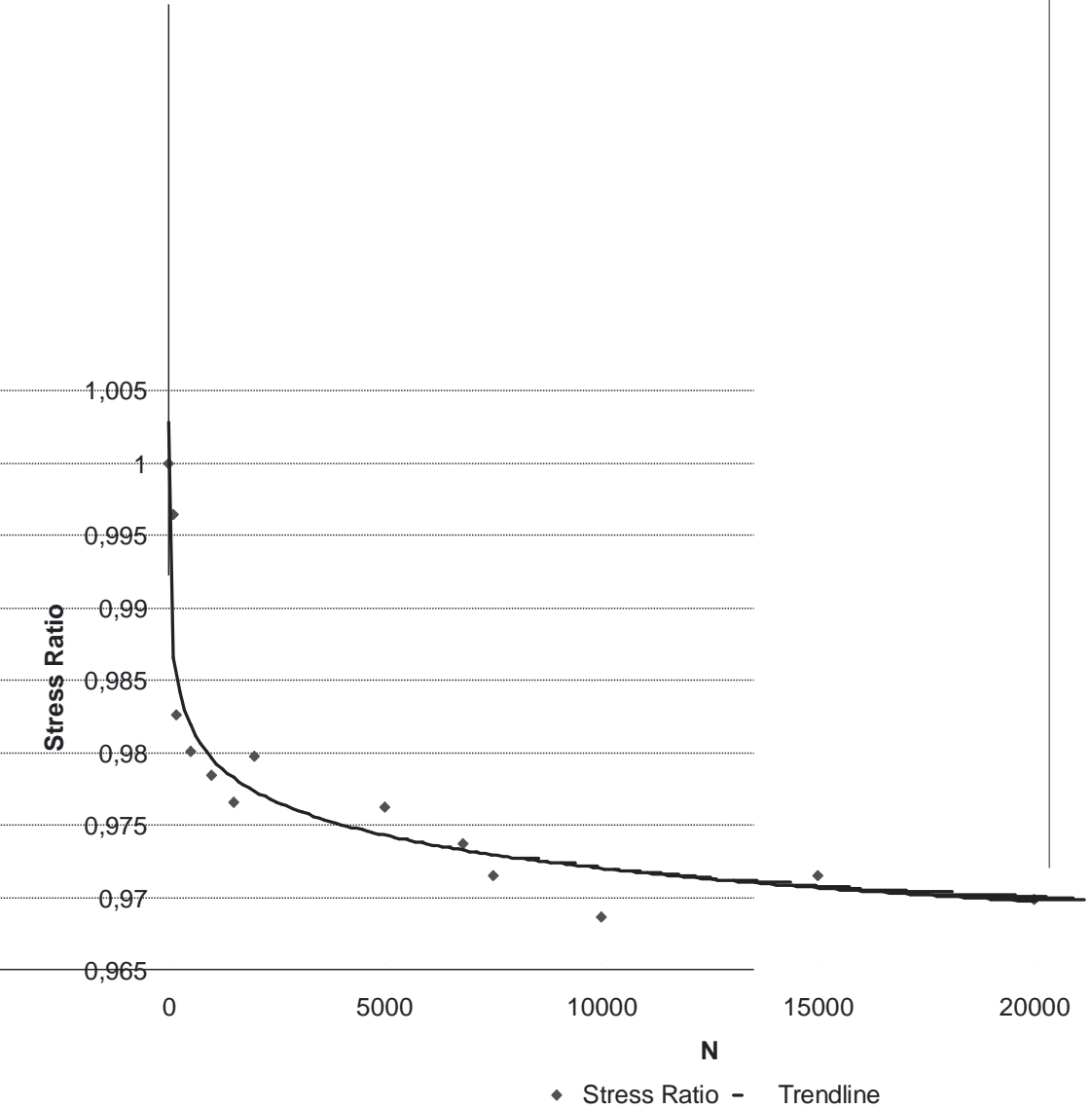

Fig. 7. Optimal simulation number.

tooth profile. These points are distributed identically for the involute and the optimized profiles. The equivalent stress at point I will still have the same strength. This is a direct consequence of the equal curvature at the pitch circle. In fact, the reduction in stress is present, most particularly, in the transition zones from one to two teeth. In our simulation result, the loading is defined with a Coulomb friction coefficient and isotorque distribution. The choice of this configuration was motivated by its restrictive and representative aspect. Indeed, the deterioration of the contact in a gear assembly increases if the contact is dry, simulating loss of the oil film, whence the choice of Coulomb friction. In the case of isotorque distribution of the load, the contact transition from one to two teeth occurs instantly and suddenly on the flank of the teeth. This provides a better picture of the stress benefits at the characteristic points A, B, C and D. The stress of the optimized profile is maximum in the vicinity of $\mathrm{I}$. In the transition zones from one to two teeth in contact, the stress is slightly diminished. The reduction in stress is of around $40 \mathrm{MPa}$ at $\mathrm{B}$ and $\mathrm{C}$, but $500 \mathrm{MPa}$ at the input (A) and $200 \mathrm{MPa}$ at the output (D). For the loading case considered, the stress benefit is some $14 \%$ during meshing.

The tooth profile minimizing the stress during meshing is obtained. The active profile of the pinion tooth differs from the conventional involute profile (Fig. 8).

Table 2 shows the values of the various coefficients for the polynomial expressions for the pinion and the wheel obtained by the tooth optimization procedure.

\section{Study of the sensitivity of the equivalent stress to geometric variations}

This study was conducted to test the robustness of the optimized profile to the geometric variations due to

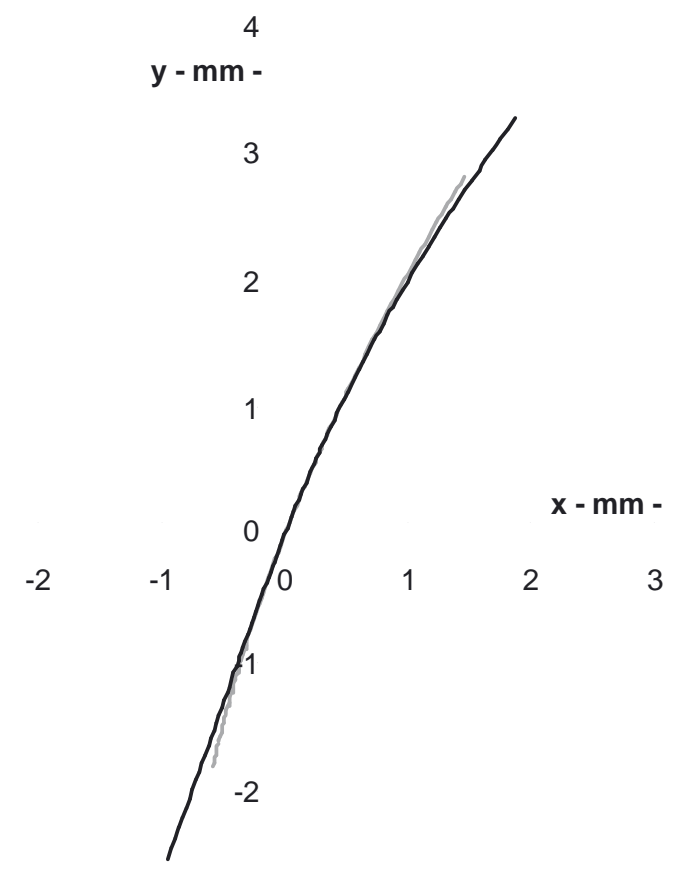

$-3$

\section{- Involute - Optimized}

Fig. 8. Involute tooth profile and optimized tooth profile.

machining imperfections. Two studies were considered at the characteristic points: propagation by Monte Carlo method and analytical propagation of uncertainties.

In the Figure 9, the localization of four characteristics points $(a, b, c$ and $d$ ) on the pinion tooth profile is represented. During the machining, these points will be perturbed by the production means. A dispersion zone of 
Table 2. Coefficients optimized tooth profile of pinion and wheel.

\begin{tabular}{lccccccc}
\hline \multicolumn{4}{c}{ Pinion coefficients } & \multicolumn{4}{c}{ Wheel coefficients } \\
\hline$C_{1 \mathrm{X}}$ & -0.591 & $C_{1 \mathrm{Y}}$ & -0.445 & $C_{1 \mathrm{X}}$ & -0.566 & $C_{1 \mathrm{Y}}$ & -0.398 \\
$C_{2 \mathrm{X}}$ & 18.041 & $C_{2 \mathrm{Y}}$ & 12.463 & $C_{2 \mathrm{X}}$ & 16.488 & $C_{2 \mathrm{Y}}$ & 11.749 \\
$C_{3 \mathrm{X}}$ & -32.028 & $C_{3 \mathrm{Y}}$ & -18.954 & $C_{3 \mathrm{X}}$ & -13.462 & $C_{3 \mathrm{Y}}$ & $1.749-$ \\
$C_{4 \mathrm{X}}$ & -51.940 & $C_{4 \mathrm{Y}}$ & -80.826 & $C_{4 \mathrm{X}}$ & -46.871 & $C_{4 \mathrm{Y}}$ & 119.486 \\
\hline
\end{tabular}

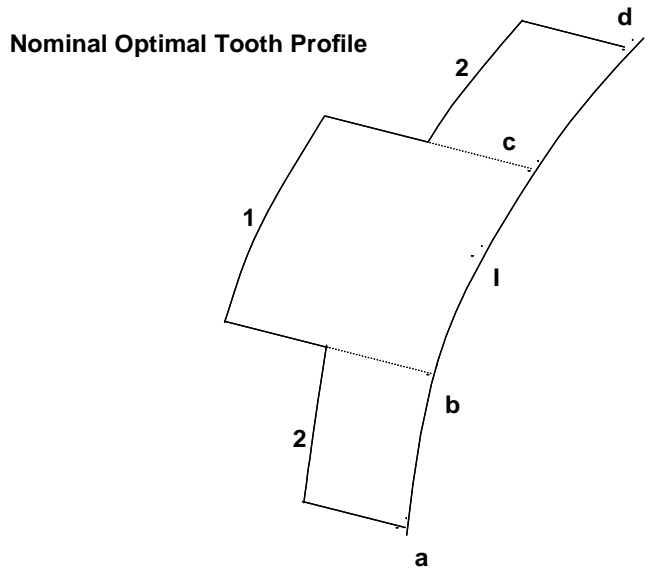

Fig. 9. Localisation of characteristics points on the tooth profile of pinion, $a, b, c$ and $d$.

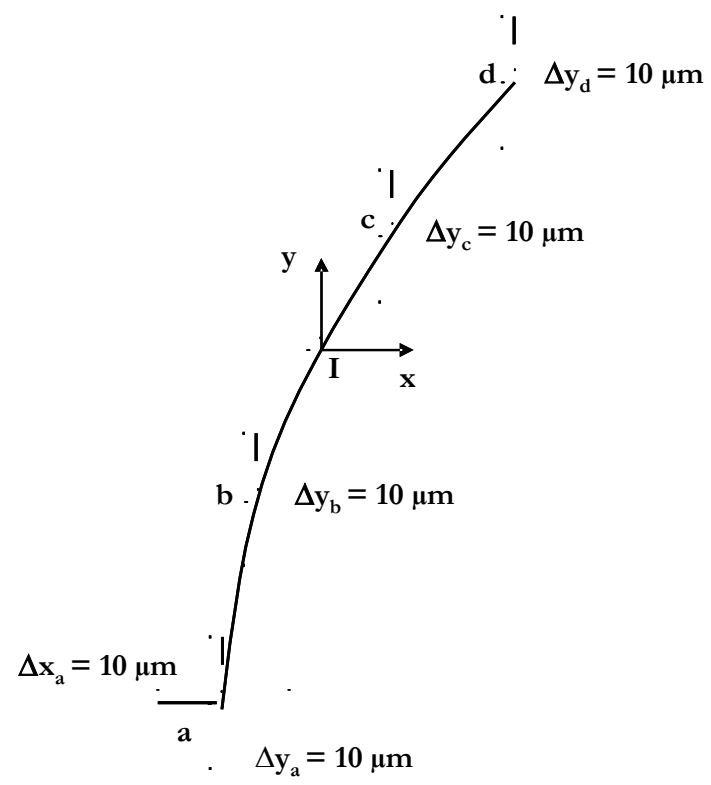

Fig. 10. Dispersion zone of manufacturing on the characteristics points.

$0.01 \mathrm{~mm}$ is represented on the flank of an optimized tooth in Figure 10. In order to re-use the polynomial expressions for the tooth profile, the choice was made to introduce this geometrical dispersion at the four characteristic points $a, b, c$ and $d$ of the tooth profile. These dispersion zones $\left(W_{\left(x_{a} ; y_{a} ; y_{b} ; y_{c} ; y_{d}\right)}=0.01 \mathrm{~mm}\right)$ applied to our two models (pinion and wheel) are defined in Figure 10.

The results of the two studies are presented in the paragraphs below.

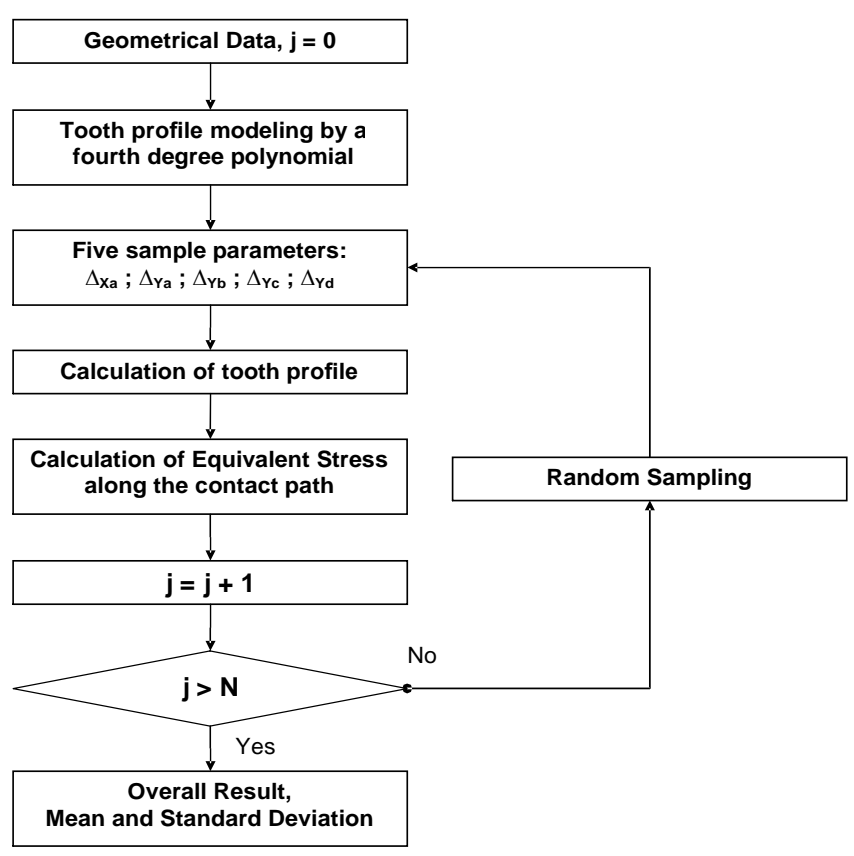

Fig. 11. Flowchart of propagation method by Monte Carlo.

\subsection{Propagation of uncertainties by Monte Carlo}

The procedure adopted to propagate the geometric uncertainties by the Monte Carlo simulation is detailed in the flowchart in Figure 11. In our study, the geometric variations were introduced at the characteristic points of the tooth profile in order to calculate the average values and the error bar at two standard deviations $(2 \sigma)$ of the maximum equivalent contact stress during meshing. To that end, the five coordinates of the previously defined characteristic points, vary randomly within the dispersion zone of manufacturing. The method also demands that all the variables are independent. This is the reason why the evolution of the geometric parameters was chosen in a Cartesian coordinate system.

The uncertainty propagation study was conducted on the optimized tooth profile. In that geometry, the variables are:

- The abscissa $X$ and ordinates $Y$ of the input contact point on the flank of the tooth: $a$.

- The ordinates $Y$ of the three other characteristic points on the flank of the tooth:

- The two points of switchover to a single point of contact: $b$ and $c$.

- The output contact point: $d$. 
Table 3. Results of the Monte Carlo simulation.

\begin{tabular}{|c|c|c|c|}
\hline Type & Stress mean & Standard deviation & 95\% confidence interval \\
\hline Uni & $1369.2 \mathrm{MPa}$ & $10.8 \mathrm{MPa}$ & $\pm 21.5 \mathrm{MPa}[1347.7 ; 1390.7]$ \\
\hline Gaussian distribution & $1368.4 \mathrm{MPa}$ & $7.0 \mathrm{MPa}$ & $\pm 14.1 \mathrm{MPa}[1354.3 ; 1382.5]$ \\
\hline
\end{tabular}

Table 4. Results of the analytical propagation.

\begin{tabular}{cccc}
\hline Type & Stress mean & Standard deviation & $95 \%$ confidence interval \\
\hline Uniform distribution & $1368.7 \mathrm{MPa}$ & $10.7 \mathrm{MPa}$ & $\pm 21.3 \mathrm{MPa}[1347.4 ; 1390.0]$ \\
Gaussian distribution & $1368.7 \mathrm{MPa}$ & $9.2 \mathrm{MPa}$ & $\pm 18.5 \mathrm{MPa}[1350.2 ; 1387.3]$ \\
\hline
\end{tabular}

Two laws of distribution were considered, one uniform and the other Gaussian. The results of these two simulations are presented in Table 3.

\subsection{Calculation by analytical propagation}

The second uncertainty propagation study was intended to confirm the results obtained via the Monte Carlo simulation. This analytical propagation method is based on G.U.M. standard [22]. The propagated uncertainty is derived from the product of the VarianceCovariance matrix (Eq. (13)) and the Jacobian of the stress function:

$$
\operatorname{Var}\left(\sigma_{\mathrm{eq}}\right)=J \operatorname{Cov}\left(x_{a} ; y_{a} ; y_{b} ; y_{c} ; y_{d}\right) J^{T}
$$

Both types of distribution were considered, i.e.: uniform (Eq. (14)) and Gaussian (Eq. (15)). The expression of the standard deviation differs for each law.

$$
\begin{aligned}
\sigma^{\left(x_{a} ; y_{a} ; y_{b} ; y_{c} ; y_{d}\right)} & =\frac{W\left(x_{a} ; y_{a} ; y_{b} ; y_{c} ; y_{d}\right)}{W\left(x_{a} ; y_{a} ; y_{b} ; y_{c} ; y_{d}\right)} \\
\sigma^{\left(x_{a} ; y_{a} ; y_{b} ; y_{c} ; y_{d}\right)} & =\frac{4}{}
\end{aligned}
$$

Equation (16) details expression 13. The independence of the variables used in this method results in a diagonal Variance-Covariance matrix. The result of this calculation is the variance of the equivalent stress. It enables the determination of the Standard Deviation $\left(\sigma_{\text {eq }}\right)$ of the equivalent stress during meshing according to the five chosen simulation parameters.

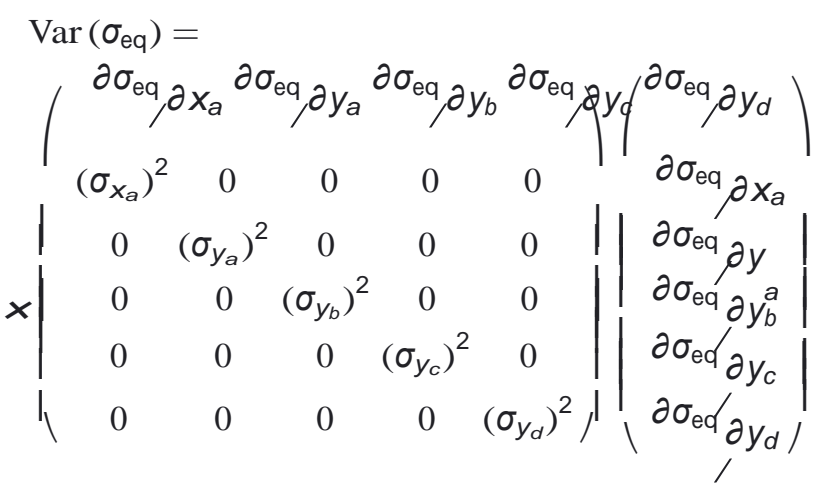

The results obtained by analytical propagation are presented in Table 4.

\subsection{Discussion of the results}

By studying Tables 3 and 4, for both types of distribution considered: uniform and Gaussian, close similarities were found. The degree of uncertainty were calculated for a risk of $5 \%$. The results obtained for the degree of error of the maximum equivalent stress was small (less than $1.5 \%$ of the mean value). This shows the low sensitivity of the equivalent stress to geometric variations (machining imperfections, wear, etc.). The results from the two methods prove the robustness and local linearity of the geometric definition of the optimum tooth profile for the evaluation criterion adopted.

\section{Conclusion}

The new mechanical requirements for gears compel designers to propose new innovative tooth geometries. These geometries must meet service life criteria under ever more demanding loads. Through this paper, our work was aimed at two objectives:

- to propose a method for optimizing the tooth profile by equivalent stress,

- to guarantee the suitability and the robustness of the profile at manufacturing variations.

The design tool proposed is a methodology which converges towards an optimum tooth profile. The overall dimensions and the loading of the gear are input data. The approach shows the existence of profiles, other than involute, which provide a useful response for increasing the service life of gearboxes. This method agrees with the work already undertaken on the geometric modification of tooth profiles, such as that by Litvin [13] in particular. The method presented in this paper thus enables identification of a geometric tooth profile in a given operating configuration. This tool, which is an aid for gear design, provides an original approach for the geometric design of a reduction gear. The gear adopted provides better behavior than an involute tooth profile for a given loading.

The study of the propagation of geometric errors concludes on the robustness of the equivalent stress with respect to the capability of the manufacturing means. The propagation behavior is locally linear. In other words, the stress variation during meshing is due to factors other than simply the geometric design of the flank of the teeth. 
Indeed, the dispersion of the characteristic points has negligible effect on the stress variations. Monte Carlo Simulation and Analytical Propagation enabled evaluation of the influence of these geometrical deviations.

The conclusions of this paper show that there is a viable and credible alternative to the involute tooth profile.

\section{References}

[1] G. Henriot, Engrenages - Conception - Fabrication Mise en œuvre - Ed. DUNOD (2007), $8^{\mathrm{e}}$

[2] ISO Standard 21771:2007(E), Gears - Cylindrical involute gears and gear pairs - Concepts and geometry, International Organization for Standardization, Geneva, Switzerland, 2007

[3] V. Spitas, T. Costopoulos, C. Spitas, Fast modeling of conjugate gear tooth profiles using discrete presentation by involute segments, Mech. Mach. Theory 42 (2007) 751-762

[4] G. Ye, X.Y. Ye, A new method for seeking the optimum gear tooth profiles - the theoretical basis of WildhaberNovikov gearing, Mech. Mach. Theory 37 (2002) 10871103

[5] V.V. Simon, Influence of tooth modifications on tooth contact in face-hobbed spiral bevel gears, Mech. Mach. Theory 46 (2011) 1980-1998

[6] M. Faggioni, F.S. Samani, G. Bertacchi, F. Pellicano, Dynamic optimization of spur gears, Mech. Mach. Theory 46 (2011) 544-557

[7] P. Velex, J. Bruyère, D.R. Houser, Some analytical results on transmission errors in narrow-faced spur and helical gears: Influence of profile modifications, ASME J. Mech. Desig. 133 (2011) 31010-31010

[8] D. Ghribi, J. Bruyère, P. Velex, M. Octrue, M. Haddar, A contribution to the design of robust profile modifications in spur and helical gears by combining analytical results and numerical simulations, ASME J. Mech. Des. 134 (2012) 61011-61011

[9] J.I. Pedrero, M. Pleguezuelos, M. Artés, J.A. Antona, Load distribution model along the line of contact for involute external gears, Mech. Mach. Theory 45 (2010) 780794

[10广 T. Osman, P. Velex, A model for the simulation of the interactions between dynamic tooth loads and contact fatigue in spur gears, Tribol. Int. (2011) 84-96

[11]J. Pedrero, M. Pleguezuelos, M. Munoz, Contact stress calculation of undercut spur and helical gear teeth, Mech. Mach. Theory 46 (2011) 1633-1646
[12] S.C. Hwang, J.H. Lee, D.H. Lee, S.H. Han, K.H. Lee, Contact stress analysis for a pair of mating gears, Math. Comput. Modelling, 2011

[13] F.L. Litvin, I. Gonzales-Perez, A. Fuentes, K. Hayasaka, K. Yukishima, Topology of modified surfaces of involute helical gears with line contact developed for improvement of bearing contact, reduction of transmission errors, and stress analysis, Math. Comput. Model. 42 (2005) 10631078

[14] J.M. Linares, J.M. Sprauel, S. Aranda, P. Bourdet, Impact of geometric uncertainties onto the operating performance of a mechanical system, in: J.K. Davidson (Ed.), models for computer aided tolerancing in design and manufacturing, Tempe, Arizona, USA, 2005, pp. 225-234

[15] L. Zamponi, E. Mermoz, J.M. Linares, J.M. Sprauel, Impact of geometrical defects on bearing assemblies with integrated raceways in aeronautical gearboxes, Mech. Mach. Theory 44 (2009) 1108-1120

[16] L. Zamponi, E. Mermoz, J.M. Linares, Étude des méthodes de calcul des pressions de contact dans les roulements à pistes intégrées des boites de transmission aéronautiques, Mécanique Industries 8 (2007) 567-576

[17] L. Zamponi, E. Mermoz, J.M. Linares, Contact pressure calculation methodologies in aeronautic gearboxes in the CAD process. The future of product development: proceedings of the 17th CIRP design conference, 2007, pp. $451-462$

[18] H. Xu, A. Kahraman, N.E. Anderson, D.G. Maddock, Prediction of mechanical efficiency of parallel-axis gear pairs, ASME J. Mech. Des. 129 (2007) 58-68

[19] J.M. Linares, J.M. Sprauel, P. Bourdet, Uncertainty of reference frames characterized by real time optical measurements: Application to computer Assisted Orthopaedic Surgery, CIRP Annals - Manuf. Technol. 58 (2009) 447-450

[20] J. Bruyère, J-.Y. Dantan, R. Bigot, P. Martin, Statistical tolerance analysis of bevel gear of tooth contact analysis and Monte Carlo simulation, Mech. Mach. Theory 42 (2007) 1326-1351

[21] D.A. Hills, D. Nowell, A. Sackfield, Mechanies of Elastic Contacts, Butterworth - Heinemann Ltd (Oxford), 1993

[22] Guide to the expression of uncertainty in measurement, (1993), 1st Edition, International Organization for Standardization (I.S.O.) 